\title{
Talker Identification using Reflection Coefficients
}

\author{
K. Eguchi, Y. Mochizuki, M. Taniguchi, K Nishida, and S. Sugimoto \\ Department of Electrical and Electronic Engineering, Ritsumeikan University \\ Noji-Higashi, Kusatsu City, Shiga 525-8577 Japan \\ Tel: +81-(0)77-561-2673, FAX: +81-(0)77-561-2663 \\ e-mail: sugimoto@se.ritsumei.ac.jp
}

\begin{abstract}
In this paper, we propose a method of identifying the talker based on modeling the voice for reading free sentences by $\mathrm{AR}$ (auto regressive) models. By remarking the similarity of the AR models and the no loss sound-tube models for vocal tracts, we assume the features of the talker voice are the higher reflection coefficients of AR models. In the simulation, there are 6 talkers' voice as speech examples from "Japanese speech sound database of ATR." As a result, we get that average recognition rate is $90.67 \%$.
\end{abstract}

\section{Introduction}

In the recent years, information and communication technology has been developed. And, we asked our personal information. So, it is necessary for personal identification systems to be high reliability and convenience. But, sometimes we forgot some passwords, or lost some keys. So these systems are certified to use our physicals. Today, many kind of systems are running around the world. For example, vein authentication system is run in many banks. And, retinal authentication system is run in some offices.

In this study, we pay $\mathrm{AR}$ (auto regressive) models attention to what can model a vocal tract and devises a system specifying an individual by the difference of the vocal tract.

\section{Audio signal processing}

We apply AR models to input audio signal for modeling of vocal tract. And, we use burg method to presume AR parameter. However, it is assumed that vocal tract are the no loss sound tubes.

\subsection{AR model}

The AR models are used in time series analysis, to describe stationary time series. These models represent time series that are generated by passing the white noise through a recursive linear filter. The output of such a filter at the moment is a weighted sum of $\{t\}$ previous values of $\{m\}$ the filter output. The integer parameter $\{m\}$ is called the order of the AR models.

The AR models of a random process $\left\{x_{t}\right\}$ in discrete time $\{t\}$ are defined by the following expression:

$$
x_{t}=\sum_{i=1}^{m} a_{i}^{(m)} x_{t-i}+e_{t}
$$

where $\left\{e_{t}\right\}$ are zero mean Gaussian white noises.

\subsection{Burg method}

Burg method is well-known as a AR models parameter estimation method which estimates model parameters recursively toward to the higher order AR models as follows,

$$
k_{m}=\frac{-2 \sum_{t=m}^{N-1} e_{t}^{(m-1)} r_{t-1}^{(m-1)}}{\sum_{t=m}^{N-1}\left\{\left[e_{t}^{(m-1)}\right]^{2}+\left[r_{t-1}^{(m-1)}\right]^{2}\right\}}
$$




$$
\begin{aligned}
& a_{i}^{(m)}=a_{i}^{(m-1)}+k_{m} a_{m-i}^{(m-1)} \\
& e_{t}^{(m)}=e_{t}^{(m-1)}+k_{m} r_{t-1}^{(m-1)}
\end{aligned}
$$

$$
\begin{aligned}
r_{t}^{(m)} & =r_{t-1}^{(m-1)}+k_{m} e_{t}^{(m-1)} \\
e_{t}^{(0)} & =r_{t}^{(0)}=x_{t} \\
\sigma_{m+1}^{2} & =\left(1-k_{m+1}^{2}\right) \sigma_{m}^{2}
\end{aligned}
$$

where $\left\{k_{m}\right\}$ are reflection coefficients, and $\left|k_{m}\right| \leq 1$. $\left\{e_{t}^{(m)}\right\},\left\{r_{t}^{(m)}\right\}$ are forward and backward prediction errors of $m$ th order.

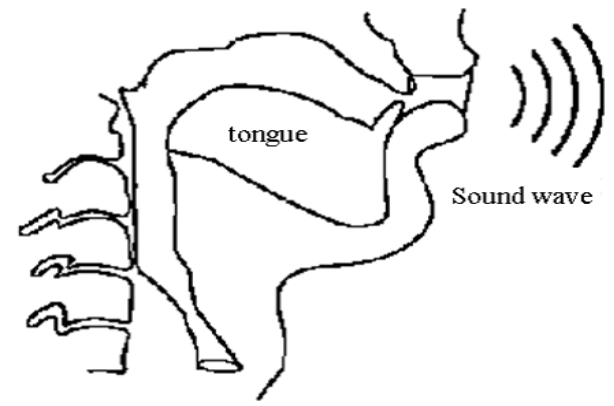

Figure 1: Structure of human throat

\section{$2.3 \quad$ Vocal tract models}

A human vocal tract is regarded as a series of sound tubes in Figs. 1 and 2, which characterize the personal voice. It is well known that the structure of the vocal tract can be assumed as the lattice filter, when sound waves travel through the tract. Forward and backward prediction errors of AR models also consist of the lattice filter which can be approximated by the vocal tract model $[3,4,5]$ (Figs. 3 and 4 ). The number of sound tubes must be equal to the order of the AR model by fitting each personal tract model to the AR model.

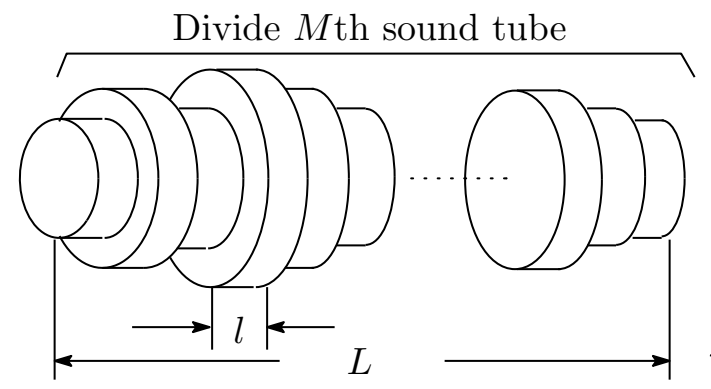

Figure 2: Vocal tract model

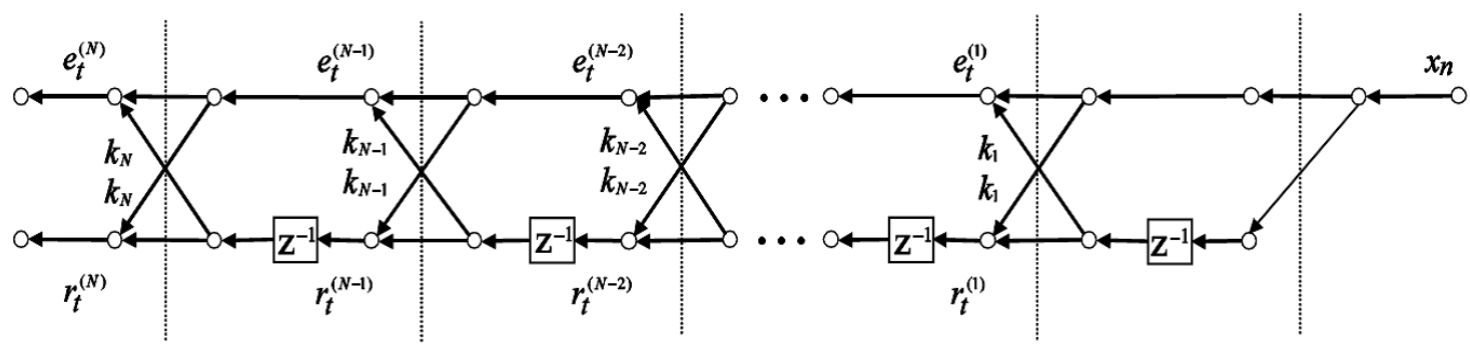

Figure 3: Lattice structure of AR models

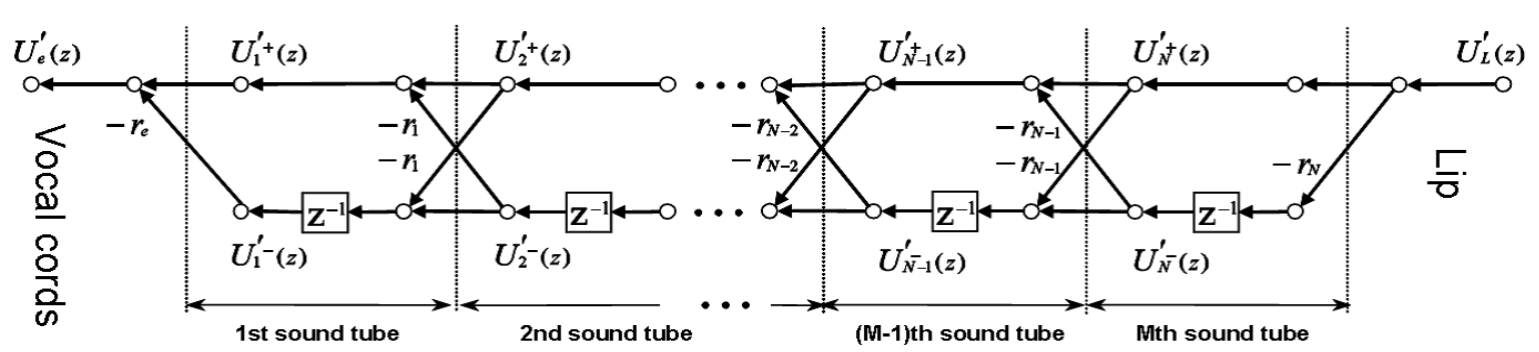

Figure 4: Lattice structure of the no loss sound tube for vocal tracts 


\section{Talker Identification}

"Talker identification" is the function of recognizing who talks. Any people has difference in his/her voice, and listener can identify who the talker is by recognizing those differences. The proposed talker identification method in this paper is utilizing the difference of reflection coefficients which shows the personal vocal tract. Reflection coefficients describe the characteristics of AR models which can be assumed as same as the vocal tract model. Therefore it is appropriate to utilize the reflection coefficients for the system in parallel processing of talker identification. Here we show the proposed talker identification method.

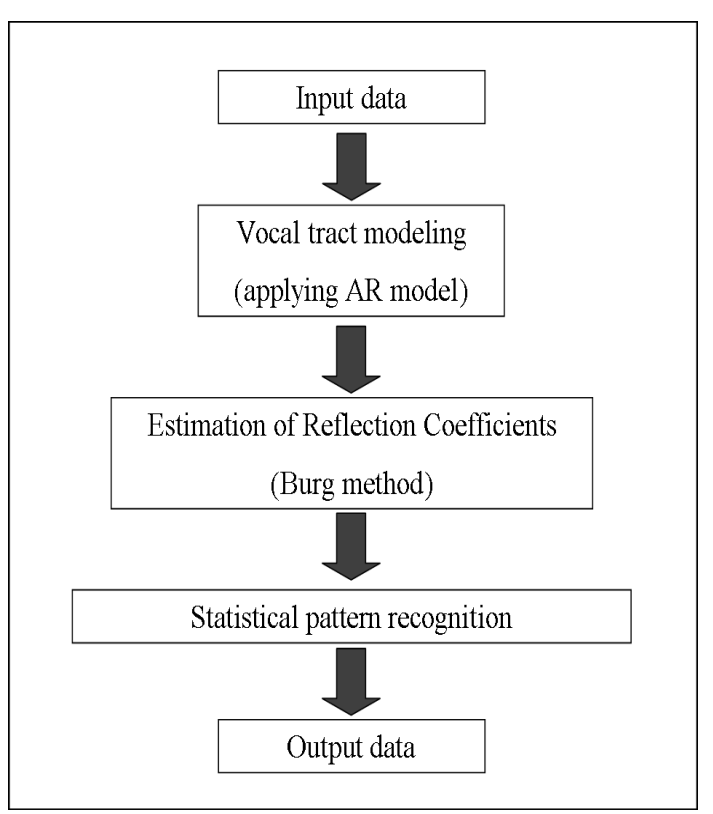

Figure 5: Flowchart of proposed talker identification

\subsection{Statistical pattern recognition}

Statistical pattern recognition $[7,8]$ is one of pattern classification methods which minimize means of grouping loss by utilizing probabilistic nature of pattern sets. In this paper, the discriminant function, which is utilized for pattern

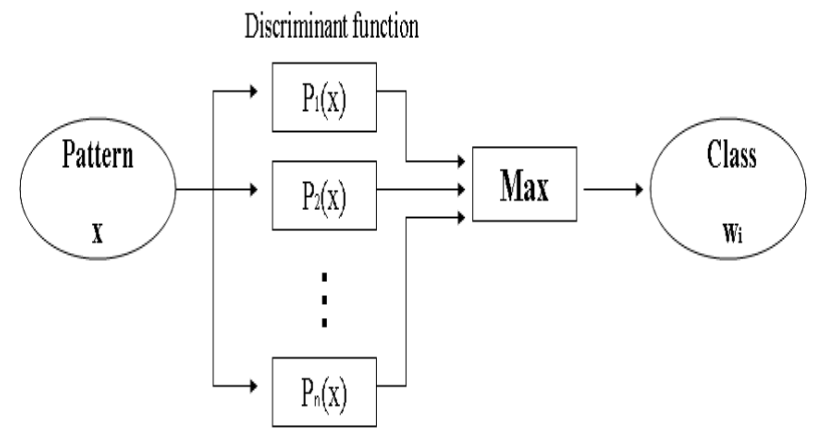

Figure 6: Process of statistical pattern recognition using discriminant functions

classification of inputs, is applied to the recognition.

\subsection{Discriminant Function}

Th probability density function (PDF) is applied as the discriminant function of the feature quantity. In our the recognition problem, the PDFs are assumed as the multidimensional normal distribution. First of all, using the test personal vocal data, we estimatethe $k$ dimentional vector of reflection coefficients $X_{q}$ for each person $q$. Then we have the PDF for the person $q$ as the following $k$-dimentional normal distribution:

$$
p_{q}(x)=\frac{1}{(2 \pi)^{\frac{k}{2}}\left|\operatorname{det} R_{q}\right|^{\frac{1}{2}}} \exp \left(-\frac{1}{2}\left(x-\mu_{q}\right)^{\mathrm{T}} R_{q}^{-1}\left(x-\mu_{q}\right)\right)
$$

where

$$
\begin{gathered}
x=\left[\begin{array}{c}
x_{1} \\
x_{2} \\
\vdots \\
x_{k}
\end{array}\right], \quad \mu_{q}=E\left[X_{q}\right]=\left[\begin{array}{c}
\mu_{q, 1} \\
\mu_{q, 2} \\
\vdots \\
\mu_{q, k}
\end{array}\right] \\
R_{q}=E\left\{\left(X_{q}-\mu_{q}\right)\left(X_{q}-\mu_{q}\right)^{\mathrm{T}}\right\} \\
=\left[\begin{array}{ccc}
\gamma_{q, 11} & \cdots & \gamma_{q, 1 k} \\
\vdots & \ddots & \vdots \\
\gamma_{q, k 1} & \cdots & \gamma_{q, k k}
\end{array}\right]
\end{gathered}
$$

$$
\left.\gamma_{q, i j}=E\left[\left(X_{q, i}(\omega)-\mu_{q, i}\right)\left(X_{q, j}(\omega)-\mu_{q, j}\right)\right] 11\right)
$$




$$
(i, j=1,2, \cdots, k)
$$

where $\mu_{q}$ stands for mean vector of $X_{q}$, and $R_{q}$ stands for covariance matrix of $X_{q}$.

\subsection{Discriminant functions applying talker identification}

The vocal data are splited by 0.01 seconds interval. We assume that the PDF of the re-

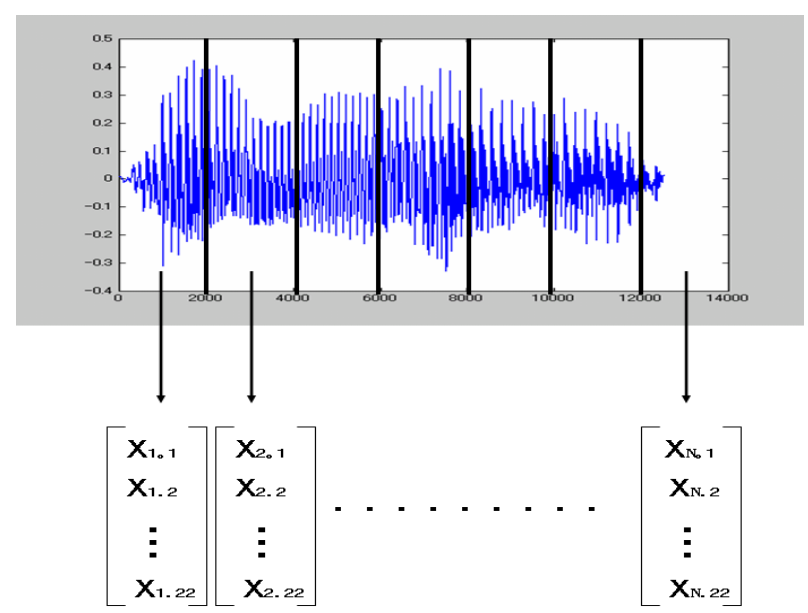

Figure 7: Fugure of spliting data

flection coefficients for each person has same PDF for each splited data. Then the likelihood function for the person $q$ of the given $N$ splitted voical data is described by

$$
\begin{aligned}
\prod_{i=1}^{N} p_{q}\left(x_{i}\right) & =\prod_{i=1}^{N} \frac{1}{(2 \pi)^{\frac{k}{2}}\left|\operatorname{det} R_{q}\right|^{\frac{1}{2}}} \\
& \exp \left(-\frac{1}{2}\left(x_{i}-\mu_{q}\right)^{t} R_{q}^{-1}\left(x_{i}-\mu_{q}\right)\right)
\end{aligned}
$$

$$
\begin{gathered}
x_{i}=\left[\begin{array}{c}
x_{i, 1} \\
x_{i, 2} \\
\vdots \\
x_{i, N}
\end{array}\right], \mu_{q}=E\left[X_{q}\right]=\left[\begin{array}{c}
\mu_{q, 1} \\
\mu_{q, 2} \\
\vdots \\
\mu_{q, N}
\end{array}\right] \\
R_{q}=E\left\{\left(X_{q}-m_{q}\right)\left(X_{q}-m_{q}\right)^{\mathrm{T}}\right\} \\
=\left[\begin{array}{ccc}
\gamma_{q, 11} & \cdots & \gamma_{q, 1 k} \\
\vdots & \ddots & \vdots \\
\gamma_{q, k 1} & \cdots & \gamma_{q, k k}
\end{array}\right]
\end{gathered}
$$

$$
\gamma_{q, i j}=E\left[\left(X_{q}-\mu_{q}\right)\left(X_{q}-\mu_{q}\right)\right]
$$

$$
(i, j=1,2, \cdots, k)
$$

\subsection{Decrease computational complex- ity}

We consider to derive the discriminant of the pattern classification system for simplification of computational cost [9]. Thus, we takes the logarithm of the equation (12) and only use the $d$ reflection coefficients of $k_{N}, k_{N-1}, \cdots k_{N-d+1}$ in Fig. 3 as feature quantities of the classification. Namely

$$
\begin{aligned}
& \sum_{i=1}^{N}\left(-\ln (\sqrt{2 \pi})^{d}-\frac{1}{2} \ln \left|\operatorname{det} R_{q}\right|-\frac{1}{2}\left(x_{i}-\mu_{q}\right)^{\mathrm{T}} R_{q}^{-1}\left(x_{i}-\mu_{q}\right)\right) \\
= & -N d \ln \sqrt{2 \pi}-\frac{N}{2} \ln \left|\operatorname{det} R_{q}\right|-\frac{1}{2} \sum_{i=1}^{N}\left(x_{i}-\mu_{q}\right)^{\mathrm{T}} R_{q}^{-1}\left(x_{i}-\mu\right)
\end{aligned}
$$

where $-N d \ln \sqrt{2 \pi}$ is constant in any case. Therefore, the discriminant function is given by

$D_{q} \equiv-N \ln \left|\operatorname{det} R_{q}\right|-\sum_{i=1}^{N}\left(x_{i}-\mu_{q}\right)^{\mathrm{T}} R_{q}^{-1}\left(x_{i}-\mu_{q}\right)$

Therefore the talker identification is done by

$$
\max _{q} D_{q}
$$

\section{Experimental Results}

Experimental results of the present talker identification method are shown for verifing the recognition rate. The Burg method is applied to speech sounds for estimating the vocal tract modelas the AR model. The statistical pattern recognition with the discriminant function in (17) has been carried out to identify the differences of reflection coeffients for the talker identification. Sample speech data are wellbalanced phoneme speech examples of man's voice in ideal environment (low-noise) from "Japanese speech sound database of ATR[10]" (Some of 
them are shown in Appendices A and B). There are 6 talkers' voice as speech examples. 25 texts are used as a training set for the statistical pattern recognition, and another 25 texts are used as a test set for each talker. The simulation experiment is performed by applying Matlab ver.7.4.0. The sampling frequency is set as $22050[\mathrm{~Hz}]$, the order of AR models is 22 . Description of input data and experimental results are shown below.

Table 1: Speech data

\begin{tabular}{|c||c|}
\hline Number of talker & 6 \\
\hline Number of input data by each talker & 50 \\
\hline Number of leaning data & 25 \\
\hline Number of recognition data & 25 \\
\hline Sampling frequency[Hz] & 22050 \\
\hline Bit rate[kbps] & 320 \\
\hline One intput data contains words & $20 \sim 75$ \\
\hline Number of frame & $125 \sim 700$ \\
\hline
\end{tabular}

Table 2: Experimental results of the talker identification using reflection coeffients

\begin{tabular}{|c||c|c|}
\hline talker & correct[\%] & incorrect[\%] \\
\hline \hline man A & 100 & 0 \\
\hline man B & 100 & 0 \\
\hline man C & 88 & 12 \\
\hline man D & 64 & 36 \\
\hline man E & 92 & 8 \\
\hline man F & 100 & 0 \\
\hline ave. & 90.67 & 9.33 \\
\hline
\end{tabular}

The recognition rates show good results of talker identification except for the man D.

\section{Conclusions and future works}

In this paper, we show that the reflection coefficients are able to utilize talker identification. By remarking that the lattice structure of the AR model corresponds to the structure of the human vocal tract, we derive a discriminant function consist of the mean and covariance matrices of the reflection coefficients. Therefore the proposed talker identification method does not depend on kinds of speech words, but depend on the personal vocal tract.

The experimental results have shown the high recognition rate except for one person. The experimental results show some usefulness of the present talker identification method. But we should consider more deeply the recognition rate on the man D. Furthermore the future works are: 1 ) simulation with wide-variety speech examples, 2) simulation under some type of noises. In the experiment, there are not enough speech examples (only 6 men's speech examples) under the low noise.

\section{References}

[1] K. Nakamuro, K. Haruki, and S. Sugimoto. The KanNon System - Real Time Speech Visualization. Internatinal Journal of Innovative Computing, Information $\&$ Control, Vol. 1, No. 3, pp. 561-547, September (2005).

[2] K. Nakamuro, M. Ishi, K. Haruki, and S. Sugimoto: The KanNon System - Real Time Visualization of Speech. Proc. of The 36th ISCIE International Symposium on Stochastic Systems Theory and Its Applications, pp. 271-276, November, (2004).

[3] K. Nakamuro, T. Wada, and S. Sugimoto. An AR Model Identification Method using a Prior Information and Its Application to a Spectral Estimation of Speech Signal (in Japanese), Trans. of the Institute of Systems, Control and Infornmation Engineers, Vol. 18, No. 5, pp. 171-177, (2002).

[4] T. Kitamura, and P. Mokhtari. Effects of voweltypes on perception of speaker characteristics of unknown speakers. Proceedings of the 2006 RISP International Workshop on Nonlinear Circuits and Processing (NCSP' 06), pp. 45-48, (2006) 
[5] H. Takemoto, K. Honda, S. Masaki, Y. Shimada, and I.Fujimoto. Measurement of temporal changes in vocal tractarea function during a continuous vowel sequence using a 3Dcine-MRI technique. Proc. the 6th International Seminaron Speech Production, pp. 284-289 (2003).

[6] J. P. Burg: Maximum Entropy Spectral Aanlysis. Ph. D Dissertation, Stanford Univ., Stanford, CA, (1975).

[7] K. Fukunaga. Introduction to Statistical Pattern Recognition Second Edition, Academic Press, NewYork (1990)

[8] T. Soeda, M. Ota, and S. Omatu. Fundamentals and Application of Mathematical Principle Statistics. Nissin-Syuppan, Tokyo (1980), (in Japanese).

[9] D. O'Shaughnessy: Speech Communications Human and Machine Second Edition, IEEE Press (2000)

[10] H. Kuwabara, Y. Sagisaka, K. Takeda, and M. Abe. Construction of ATR Japanese Speech Database as A Research Tool. Technical Report TR-I-0086, ATR, (1989), (in Japanese).

\section{A Example of training sets data}

- Arayuru gennzitu wo subete jibunn no hou e nejimageta no da.

- 1 syuukann bakari New York wo syuzai sita.

- TV game ya PC de game wo site asobu.

\section{B Example of test set data}

- Miageru fuji mo iiga rojuue mata hatiue no hana mo kirei desu.

- Haha wa mokessenn no kouisyou de roujinnnsei tihousyou ni nari 1 nenn mae kara nyuuinn tyuu desu.

- Pajama to T syatu ga mekurete usui rokkotu no sita ni petyannko no hara ga mieta. 